\title{
Multi-Objective Reactive Power Optimization Including Distributed Generation
}

\author{
WANG Hong ${ }^{1,2}$, WANG Zhijie ${ }^{1}$ \\ 1. Shanghai Dianji University, Shanghai, China \\ 2. University of Strathclyde, England, UK \\ E-mail:wzjsdstu@163.com
}

\begin{abstract}
In order to solve the problem of reactive power optimization of distribution network with distributed power supply, the multi-objective reactive power optimization function is established from multiple perspectives, and the equation constraint and inequality constraint equation of power system are considered. Secondly, taking IEEE33 node distribution system with distributed power supply as an example, reactive power optimization of single objective function is carried out to verify that the proposed algorithm has a global convergence and a great advantage in convergence speed. Finally, multi-objective reactive power optimization of distribution network with distributed power supply is carried out. Simulation results demonstrate the effectiveness of the proposed algorithm. Keywords: Distributed generation; Multi-objective optimization; Active distribution network; Modeling and simulation
\end{abstract}

\section{Introduction}

The traditional reactive power optimization of distribution network is mainly from the economic point of view[1-5]. However, single-objective optimization will ignore the interests of other aspects, and different factors need to be taken into account in the decisions of DG investment subjects. The power sector or DG investors are more eager to get the maximum benefits, and they pay more attention to the investment cost of DG. As users, they hope to obtain safe and stable electricity, so they mainly focus on the quality of power. It can be seen that under the multiple requirements of power network operation quality and economic benefits, the future direction of reactive power optimization is to comprehensively consider the economy, safety and power supply reliability of power system operation[6-9], so it is necessary to establish a multi-objective reactive power optimization model to meet the needs of multiple parties.

\section{Reactive power optimization mathematical model of active distribution network with DG}

\subsection{Establishment of reactive power optimization model}

This article from the perspective of economy, power supply safety and operation cost, establish the minimum system active network loss and voltage deviation of minimum investment cost and minimum multi-objective reactive power optimization function model, flow equation for power systems at the same time consider equality constraints and inequality constraints, the use of the proposed improved MOMTLBO(Improved Multi-Objective teaching-learning-based Optimization algorithm, MOMTLBO)[10]IEEE33 node containing DG system for simulation analysis.

1) System active network loss

Economy is often an important factor to be considered in reactive power optimization. The specific mathematical expression of the system active power network loss is shown in equation (1).

$$
\min f_{\text {loss }}=\sum_{i, j \in N_{B}} G_{i j}\left(U_{i}^{2}+U_{j}^{2}-2 U_{i}^{2} U_{j}^{2} \cos \theta_{i j}\right)
$$

In the formula, quantities are defined as: $f_{\text {loss }}$ is system active network loss; $N_{B}$ is number of system nodes; $U_{i}$ is the voltage at node $i ; G_{i j}$ is admittance between nodes $i$ and $j ; \theta_{i j}$ is the phase angle difference between nodes $i$ and $j$.

2) Node voltage deviation

As one of the important indexes to measure the power quality, voltage will directly affect the performance of electrical equipment, and one of the important purposes of reactive power optimization is to improve the power 
quality as much as possible[11]. In this paper, the deviation between node voltage and standard voltage is selected as one of the objective functions. The node voltage deviation formula is shown in equation (2).

$$
\min f \Delta U=\sum_{i=1}^{N}\left(\frac{U_{i}-U_{e}}{U_{i, \max }-U_{i, \min }}\right)^{2}
$$

In the formula, quantities are defined as: $f \Delta U$ is distribution network node voltage deviation; $N$ is number of distribution network nodes; $U_{e}$ is the expected voltage value of the node; $U_{i, \max }$ is nodal voltage upper limit; $U_{i, \min }$ is node voltage lower limit.

3) Cost optimization

From the perspective of power companies or DG investors, reactive power optimization cost should be considered as low as possible, so it is necessary to take DG optimization cost as one of the objective functions. The mathematical expression of DG optimization cost is shown in equation (3).

$$
\min f_{Q}=\sum_{n \in N_{C}} C_{\text {capn }}\left|P_{n}\right|
$$

In the formula, quantities are defined as: $f_{Q}$ is DG optimization cost; $P_{n}$ is investment cost per unit capacity of node $\mathrm{n}$; $C_{\text {capn }}$ is the actual compensation capacity of node $\mathrm{n}$.

\subsection{Reactive power optimization constraints}

Reactive power optimization constraints of power system include two aspects: equality constraints and inequality constraints.

1) equality constraints

Equation constraint condition is power constrained power flow equation, and the specific mathematical expression is as follows:

$$
\left\{\begin{array}{l}
P_{i}=P_{G i}-P_{L i}=U_{i} \sum_{j=1}^{N} U_{j}\left(G_{i j} \cos \theta_{i j}+B_{i j} \sin \theta_{i j}\right) \\
Q_{i}=Q_{G i}+Q_{C i}-Q_{L i}=U_{i} \sum_{j=1}^{N} U_{j}\left(G_{i j} \sin \theta_{i j}-B_{i j} \cos \theta_{i j}\right)
\end{array}\right.
$$

In the formula, quantities are defined as: $P_{G i}$ and $Q_{G i}$ are active and reactive power output of generator nodes; $P_{L i}$ and $Q_{L i}$ are load consumes both active and reactive power; $Q_{C i}$ is reactive power compensation capacity; $G_{i j}$ is the conductance between node $i$ and node $j ; B_{i j}$ is the susceptance between node $i$ and node $j$; $\theta_{i j}$ is the voltage phase angle difference between node $i$ and node $j$.

2) inequality constraints

Inequality constraints include control variable constraints and state variable constraints. Constraint conditions of control variables:

$$
\left\{\begin{array}{lc}
Q_{D G j \min } \leq Q_{D G j} \leq Q_{D G j \max } & j \in N_{G} \\
T_{i \min } \leq T_{i} \leq T_{i \max } & i \in N_{T} \\
Q_{C i \min } \leq Q_{C i} \leq Q_{C i \max } & i \in N_{C}
\end{array}\right.
$$

In the formula, quantities are defined as: $Q_{D G j m i n}$ is DG injects the lower limit of reactive capacity into the distribution network; $Q_{D G j m a x}$ is DG injects the upper limit of reactive capacity into the distribution network; $T_{i m i n}$ is lower limit of tap position of load regulating transformer tap; $T_{\text {imax }}$ is upper limit of tap position of load regulating transformer tap; $Q_{\text {Cimi }}$ is tap position of load regulating transformer tap; $Q_{\text {Cimax }}$ is the lower and upper limits of the reactive capacity of a reactive compensation device;

Inequality constraints of state variables:

$$
U_{i \min } \leq U_{i} \leq U_{i \max } \quad i \in N_{l}
$$

In the formula, quantities are defined as: $U_{\text {imin }}$ is lower limit of load node voltage; $U_{\text {imax }}$ is upper limit of the load node voltage; $N_{l}$ is number of load nodes in the system.

\subsection{Reactive power optimization procedure and flow chart}

The steps of reactive power optimization are as follows: 
Step 1: Input the parameters of distribution network system to determine the compensation point for reactive power compensation; the number of classes is $C$, total number of students is $N$, the maximum number of iterations

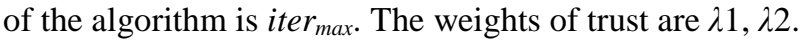

Step 2: All individuals are initialized and their objective function values are calculated, that is, the power flow of the system is calculated. Individuals were sorted according to the fast non-dominant sorting method, and 'teachers' were selected by niche technology.

Step 3: Conduct teaching according to the formula $X_{i}^{\prime}=\lambda_{1} X_{i}+$ Difference $M_{i}$, form a new class, deal with constraints on new individuals, calculate the value of objective function of all individuals, and update C;

Step 4: Learn from each other according to the formula

$$
X_{i}^{\prime \prime}=\left\{\begin{array}{l}
\lambda_{2} X_{i}^{\prime}+r_{i}\left(X_{i}^{\prime}-X_{m}^{\prime}\right) f\left(X_{i}^{\prime}\right)<f\left(X_{m}^{\prime}\right) \\
\lambda_{2} X_{i}^{\prime}+r_{i}\left(X_{m}^{\prime}-X_{i}^{\prime}\right) f\left(X_{m}^{\prime}\right)<f\left(X_{i}^{\prime}\right)
\end{array}\right.
$$

form a new class, deal with constraints on new individuals, calculate the value of objective function of all individuals, and update C;

Step 5: According to the formula $X_{i, j}^{\prime}=X_{i, j}+\operatorname{rand} \times\left(X_{H i, j}-X_{L i, j}\right)$, self-variation learning is carried out, constraint processing is carried out on new individuals, objective function value of all individuals is calculated, and $\mathrm{C}$ is updated.

Step 6: The individuals in class $\mathrm{C}$ are non-dominant sorted, and then all the non-dominant individuals in class $\mathrm{C}$ are confirmed and stored in the Pareto optimal solution set as the non-dominant particle set. The number of individuals in the Pareto optimal solution set is checked. If the number of individuals in the Pareto optimal solution set is greater than the given value, the improved crowding distance sorting strategy is used to screen until the requirements are met.

Step 7: To determine whether the maximum number of iterations is reached, go to Step2; otherwise, perform Step8.

Step 8: Finally, the Pareto solution set that meets the requirements of reactive power optimization is output.

\section{IEEE33 node simulation example}

In this paper, IEEE33 node distribution system is selected as an example of simulation calculation, and a certain amount of DG is connected at the same time. The improved algorithm MOMTLBO is used for reactive power optimization. The actual simulation tool used is MatlabR2010b, and the computer CPU is Inter CORE i3, 2G memory, and Windows 7 system.

\subsection{The simulation results}

The improved IEEE33 node system as shown in figure 1 is used for simulation analysis. With the original branch parameters unchanged, DG with active power output of $1 \mathrm{MW}$ is connected to nodes 2 and 13, which has certain reactive power compensation capacity and reactive power output capacity of $-100 \sim 500 \mathrm{kVar}$. An on-load voltage-regulating transformer $\mathrm{T}$ is connected to the node 1-2, and the transformer tap has 8 tap positions, and the upper and lower limit of the transformer ratio is [0.9,1.1]. A reactive power compensation capacitor is incorporated into each node 6 and 31, capacities are $150 \mathrm{kVar} \times 4$ and $150 \mathrm{kVar} \times 7$. The system voltage reference value is $12.66 \mathrm{kV}$, the power reference value is $100 \mathrm{MVA}$, the system load node voltage interval is $[0.93,1.07]$.

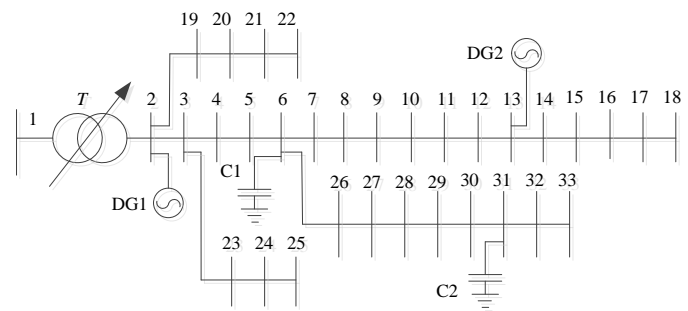

Fig.1 Diagram of IEEE33 node distribution system

\subsection{Single objective reactive power optimization of distribution network with DG}

In order to verify whether MOMTLBO(Improved Multi-Objective teaching-learning-based Optimization algorithm, MOMTLBO) has a good global optimization ability in the reactive power optimization process of power system, the single objective function with the minimum loss of active power network was established to optimize, and the optimization results were compared with the MOPSO (Multi-Objective Particle Swarm Optimization, 
MOPSO)[12] and MOTLBO(Multi-Objective teaching-learning-based Optimization algorithm, MOTLBO)[13] results. The parameter Settings of each algorithm should be consist as far as possible. The parameter Settings of each algorithm are shown in table 1.

Table1. Parameter settings for three algorithms

\begin{tabular}{llll}
\hline Algorithm & Number of iterations & Population size & \\
\hline MOPSO & 100 & 30 & wmin $=0.4$, wmax $=0.9$, c1=c2=2.05 \\
MOTLBO & 100 & 30 & - \\
MOMTLBO & 100 & 30 & $\lambda 1(\lambda \min =0.1, \lambda \max =0.6) ;$ \\
& & & $\lambda 2(\lambda \min =0.3, \lambda \max =0.8)$ \\
\hline
\end{tabular}

Fig. 2 shows the convergence characteristics curves of the three algorithms for one-shot optimization. It can be seen from the overall figure that MOMTLBO is stronger than MOTLBO and MOPSO both in terms of convergence speed and optimal solution. Although MOMTLBO converges slightly slowly at the beginning, it is obviously faster than the other two algorithms after the number of iterations reaches 10 , and it can be seen that MOPSO falls into the local optimal when the number of iterations reaches 40.

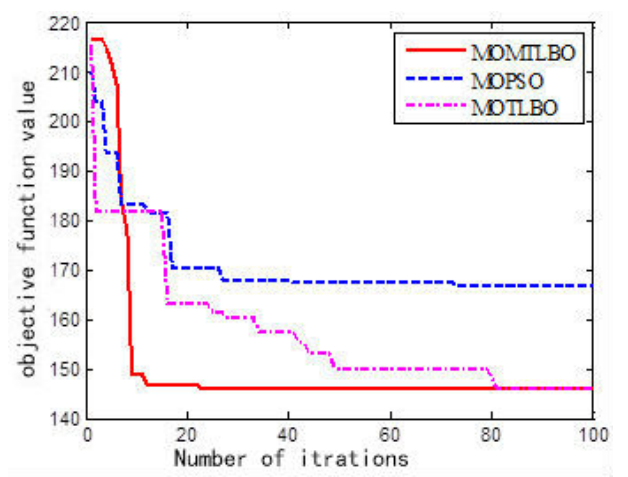

Fig 2. Comparison of convergence characteristic curves

Although the final optimization result of MOTLBO algorithm is not different from MOMTLBO, the iteration speed is much slower than that of MOMTLBO. MOMTLBO has obvious advantages and basically converges at the 22nd iteration, indicating that MOMTLBO has a strong rapidity in single-objective reactive power optimization. Table 2 shows the optimal value, the worst value and the average value of the active power network loss obtained by the three algorithms after 30 runs respectively. It can be seen from the table that the optimal values obtained by MOTLBO and MOMTLBO are significantly better than MOPSO, and the comparison of the worst values shows that MOMTLBO has a better optimization effect than MOTLBO. Before the installation of DG, the initial power flow of the system was $246.00 \mathrm{kw}$. By using the average value of the optimized algorithms, it can be concluded that the active power network loss of the system was reduced by $30.3 \%, 39.7 \%$ and $41.36 \%$ respectively after the optimization of MOPSO, MOTLBO and MOMTLBO, indicating that the improved algorithm has a good global convergence in single-objective reactive power optimization.

Table 2. Comparison after optimization

\begin{tabular}{llll}
\hline Algorithm & the optimal value & the worst value & the average value \\
\hline MOPSO & 168.72 & 179.68 & 171.47 \\
MOTLBO & 143.36 & 152.71 & 148.33 \\
MOMTLBO & 142.19 & 147.85 & 144.26 \\
\hline
\end{tabular}

\subsection{Multi-objective reactive power optimization of distribution network with DG}

MOMTLBO is used to optimize the multi-objective reactive power of the distribution network with DG. Figure 1 is still used as the simulation test system. All parameters of the system and the access to DG and reactive compensation capacitor remain unchanged. Parameters of MOMTLBO Algorithm: class size $N=100$, maximum number of iterations $T_{\max }=500$, in the weights of trust $\lambda_{1}, \lambda_{\min }=0.1, \lambda_{\max }=0.6$, in the weights of trust $\lambda_{2}, \lambda_{\min }=0.3$, $\lambda_{\max }=0.8$. Parameters of MOPSO Algorithm: particle size $N=100$, maximum number of iterations $T_{\max }=500$, inertia weight $w_{\min }=0.4, w_{\max }=0.9$, learning factors $c_{1}=c_{2}=2.05, V_{\min }=0.2 V_{\max }$.

The spatial distribution diagram of multi-objective reactive power optimization solution set in the target function is shown in Fig 3. From the graph in Fig 3 a) can be seen that the method presented in this paper optimized 
the obtained optimal solution set of even distribution, which provides decision makers with the diversity of options, the distribution of MOPSO algorithm optimization results is not uniform, part of the solution is more crowded, central shows that optimization result is very close to, the lack of diversity, will not be able to provide better choice. It shows that the improved algorithm has certain advantages. In addition, it can be concluded that many schemes are different from each other, and it is not easy to define which scheme is the best. For each optimized objective in the objective function, there are some conflicts between them and they are all related to each other. Fig.3(b-d) in the sub-fig.3 are the relationship diagram between two objective functions after the optimization of multiobjective functions by MOMTLBO algorithm. As can be seen from the figure, two of the three variables are not linear and are not independent of each other.

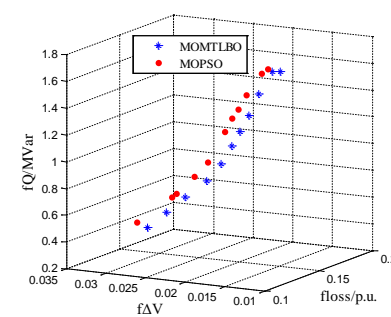

a) floss $\mathrm{f} \Delta \mathrm{V}$ and $\mathrm{fQ}$

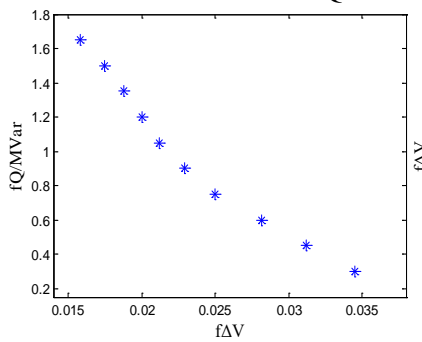

c) $\mathrm{f} \Delta \mathrm{V}$ and $\mathrm{fQ}$

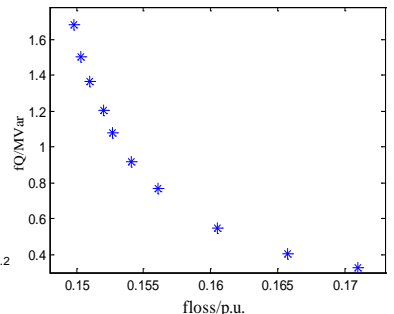

b) floss and fQ

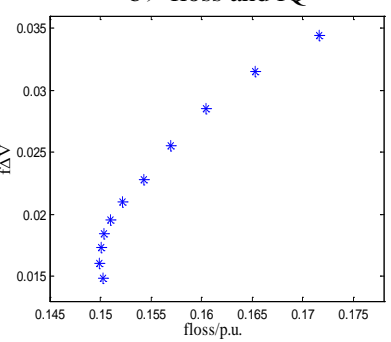

d) floss and $\mathrm{f} \Delta \mathrm{V}$

Fig 3. Pareto solutions of Reactive power optimization in the objective space

\subsection{Analysis of simulation results}

Table 3 shows the optimization scheme sets and their corresponding objective function values. As can be seen from Table 3, although there is no optimal scheme for reactive power optimization, from the perspective of decision makers, the feasible scheme can be selected if certain preferences are set. If economy is taken into consideration first, the minimum loss of active power network can be taken as the selection object. At this time, scheme S1 can be selected. If the focus is on voltage quality, the minimum voltage deviation can be taken as the selection object. At this time, scheme S4 can be selected. If the cost of reactive power optimization is expected to be the lowest, scheme S11 can be selected. When comprehensive consideration is needed, other schemes can be selected according to actual requirements.

Combined with Fig 3 and Table 3 shows that the floss, $f$ and $f Q \Delta V$ as the objective function optimization, is the contradiction between the three. According to the voltage deviation formula listed in the previous section and the circuit knowledge, the improvement of voltage not only reduces the voltage fluctuation, but also indirectly reduces the active power loss of the circuit. Fig. 4 shows S1, S5 and S9 schemes as well as the voltage curve at the time when DG is not connected. After reactive power optimization on the basis of the distribution system connected to DG, the system node voltage is effectively improved, especially the voltage at the point connected to DG is significantly increased. There are two reasons for the improvement of voltage: (1) DG has its own reactive power compensation ability and can output a certain amount of reactive power; (2) reactive power compensation equipment provides a certain amount of reactive power for the system. Within a certain range, the more reactive power the reactive compensation equipment provides, the smaller the voltage deviation and the system active power loss will be.

The network loss reduction rate and voltage deviation reduction rate of the three optimization schemes S1, S5 and S9 compared with those without access to DG and those without access to DG are shown in Table 4. It can be seen from the optimization results that the voltage deviation is greatly reduced when reactive power optimization is carried out on the basis of DG access, indicating that the access of DG improves the voltage fluctuation of the system to some extent, improves the power quality, and the active power network loss of the system is also greatly reduced. 
Table 3. Pareto solutions and simulation results of reactive power optimization

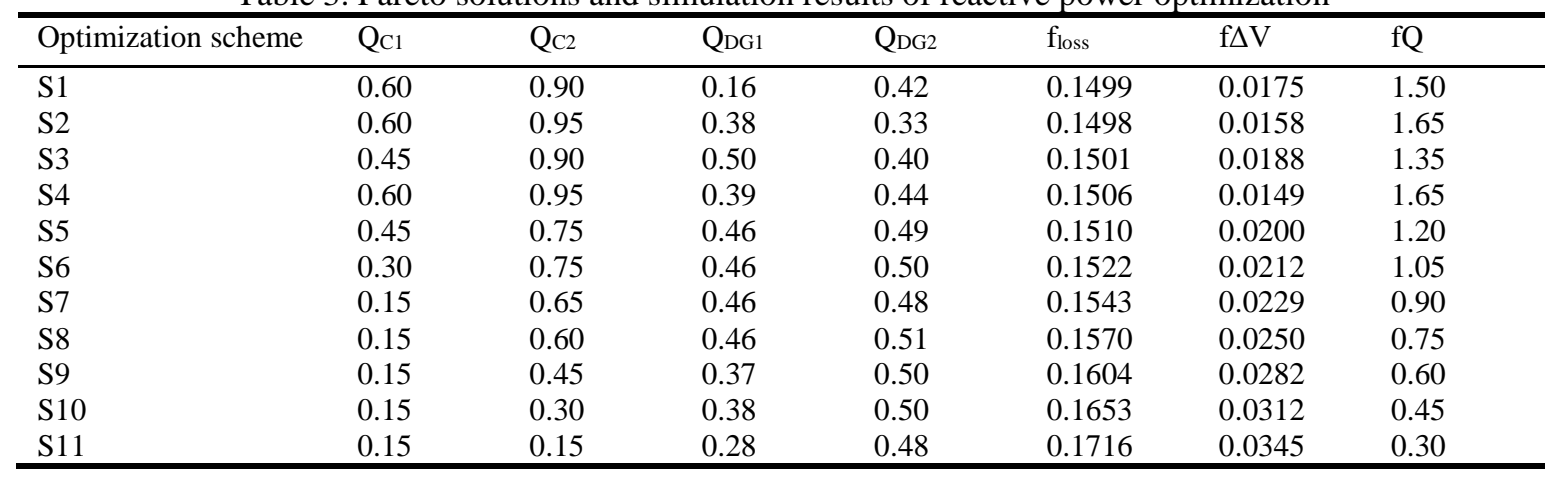

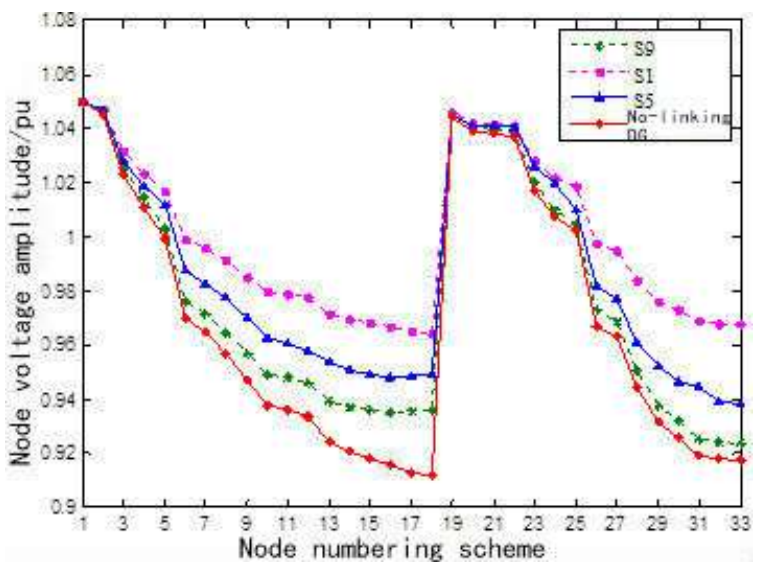

Fig 4. The influence of reactive power optimization scheme on the node voltage of the system

Table 4. Comparison of the results of the three optimization schemes

\begin{tabular}{llllll}
\hline \multirow{2}{*}{$\begin{array}{l}\text { Optimizati } \\
\text { on scheme }\end{array}$} & floss $\downarrow(\%)$ & & $\mathrm{f} \Delta \mathrm{V} \downarrow(\%)$ & \\
\cline { 2 - 3 } \cline { 5 - 6 } & Not access DG & Access to DG \& not optimized & & Not access DG & Access to DG \& not optimized \\
\hline S1 & 60.37 & 40.67 & 80.94 & 65.33 \\
S5 & 59.81 & 38.90 & 78.13 & 60.69 \\
S9 & 55.14 & 32.95 & 69.23 & 44.56 \\
\hline
\end{tabular}

\section{Conclusion}

In conclusion, in the process of multi-objective reactive power optimization, floss, $\mathrm{f} \Delta \mathrm{V}$ and fQ three objective function of the relationship between the complex, between each other is not completely independent, therefore, cannot be simply the multi-objective optimization problem by weighting method is transformed into single objective optimization problem. The algorithm proposed in this paper can obtain the diverse solution sets required by decision makers to some extent, which provides some reference value for the actual reactive power optimization of power system.

\section{Acknowledgement}

This work is supported by National Nature Science Foundation under Grant (51477099) and Key Laboratory of the Ministry of Education Foundation of China(2016AB14)

\section{References}

[1] Zhang Li, Xu Yuqin, Wang Zengping, et al. Reactive power optimization of distribution network including distributed power supply. Journal of electrotechnics, 2011,26:168-174.

[2] Xu Junjun, Huang Yonghong, Wang Qi, et al. Reactive power optimization of distribution network with DG access based on natural selection particle swarm optimization. Electrical measurement and instrumentation, 2014, 51:33-38. 
[3] Li W W, Chen J J, Duan L K, et al. Reactive power optimization based on bacterial colony optimization algorithm including distributed power supply. Electrical measurement and instrumentation,2015,52:120-124.

[4] Martinez-Rojas M, Sumper A, Gomis-Bellmunt O, et al. Reactive power dispatch in wind farms using particle swarm optimization technique and feasible solutions search. Appl Energy, 2011, 88:4678-4686.

[5] Chen Lin, Zhong Jin, Ni Yixin, et al. Reactive power optimization of distribution network including distributed generation. Power system automation,2006, 30:20-24.

[6] Malekpour A R, Tabatabaei S, Niknam T. Probabilistic approach to multi-objective Volt/Var control of distribution system considering hybrid fuel cell and wind energy sources using Improved Shuffled Frog Leaping Algorithm. Renewable Energy, 2012,39:228-240.

[7] Niknam T.A new HBMO algorithm for multiobjective daily Volt/Var control in distribution systems considering distributed generators. Appl Energy, 2011, 88:778-788.

[8] Xia Keqing, Zhao Mingqi, Li Yang. Adaptive genetic algorithm for multi-objective reactive power optimization. Power grid technology, 2006,30:55-60.

[9] Cheng Shan, Chen Minu, Huang Yichen. Research on multi-objective reactive power optimization strategy for distribution network including distributed generation. Power system protection and control,2013,41:45-50.

[10]Rao R V, Patel V. Multi-objective optimization of two stage thermoelectric cooler using a modified teachinglearning-based optimization algorithm. Engineering Applications of Artificial Intelligence, 2013;26(1):430445.

[11]Walmir Freitas, Joes C M, et al. Influence of excitation system control modes on the allowable penetration level of distributed synchronous generators. IEEE Tranctions on Energy Conversion, 2005, 20:474-480.

[12] Martinez-Rojas M, Sumper A, Gomis-Bellmunt O, et al. Reactive power dispatch in wind farms using particle swarm optimization technique and feasible solutions search. Appl Energy,2011,88:4678-4686.

[13] Rao R V, Patel V. An improved teaching-learning-based optimization algorithm for solving unconstrained optimization problems. Scientia Iranica, 2013; 20(3):710-720 